\title{
Monitoring of lignin-based polyurethane synthesis by FTIR-ATR
}

\author{
${\text { C.A. } \text { Cateto }^{a} \text {, M.F. Barreiro }}^{a, *}$, A.E. Rodrigues ${ }^{b}$ \\ a LSRE-Laboratory of Separation and Reaction Engineering, Instituto Politécnico de Bragança, \\ Campus de Santa Apolónia, Ap 1134, 5301-857 Bragança, Portugal \\ $\mathrm{b}$ LSRE-Laboratory of Separation and Reaction Engineering, Faculdade de Engenharia da \\ Universidade do Porto, Rua Dr. Roberto Frias, 4200-465 Porto, Portugal
}

\section{A R T I C L E I N F O}

Keywords:

Lignin

Polyurethanes

Synthesis

FTIR-ATR

\begin{abstract}
A B S T R A C T
FTIR-ATR (Fourier Transform Infrared Spectroscopy working in the mode of Attenuated Total Reflectance) was applied to study the formation of lignin-based polyurethanes. Although some studies related to the use of lignin in polyurethane synthesis, are available in literature, still persist a gap for a systematic study of this kind of systems, involving the measurement of kinetic data, modelling and optimization of the polymerization process. The present methodology allows the test of different formulations at a scale of 3-5 g, considering several process variables: temperature, $\mathrm{NCO} / \mathrm{OH}$ ratio, type and average molecular weight of the polyol, type of isocyanate, type and weight content of lignin.

This work aims to describe the methodology used to perform the FTIR experiments by presenting a case study. The polyurethane samples have been prepared starting with 4,4'-methylene-diphenylene isocyanate (MDI), policaprolactonediol (PCL) of three different average molecular weights (1000, 750 and 400) and a commercial lignin (Indulin AT from Meadwestvaco) at different weight contents (10, 15, 20 and 25\%). The results obtained in this work point out for the validation of the proposed experimental technique and confirm that lignin was incorporated in the final three-component polyurethane sample by chemical reaction with isocyanates, i.e., formation of urethane linkages.
\end{abstract}

() 2007 Elsevier B.V. All rights reserved.

\section{Introduction}

The synthesis of polymeric materials, using renewable resources, has been subject of research in the last decades. Many biomass components, such as, cellulose, chitin and suberine, have been used in a variety of applications (Gandini and Belgacem, 1998; Meister et al., 1992). Lignin is among these biomass components and has been the material of choice for several research groups (see Glasser and Sarkanen, 1989 and $\mathrm{Hu}, 2002$ ). The studied applications include the production of polyols, polyurethanes, acrylics, epoxy and phenolic resins. The promoting factors for the utilization of lignin as raw material for polymeric synthesis can be summarized as follows: (1) renewable and abundant raw material source. It constitutes about $15-30 \%$ of the wood and $12-20 \%$ of the annual plants; (2) material with intrinsic biodegradability. It is expected that this property will be transmitted to polymers where lignin is incorporated; (3) presence of various reactive points that can be used in a wide range of chemical reactions; (4) by-product of the pulp industry, available in large quantities (estimated in 7 million tonnes per year).

\footnotetext{
* Corresponding author. Tel.: +351 273 303000; fax: +351 273313051.

E-mail address: barreiro@ipb.pt (M.F. Barreiro). 
Table 1 - Characteristics of the materials used in the formulations

Designation

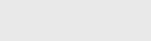

\begin{tabular}{lr}
\hline & \\
MDI & 250 \\
PCL1000 & 1000 \\
PCL750 & 750 \\
PCL400 & 400
\end{tabular}

INDULIN-AT ${ }^{\mathrm{a}}$

Molecular weight

250

1000

450
Structural formula
OH or NCO content $(\mathrm{mmol} / \mathrm{g})$

** Based on values supplied by Solvay Interox.

${ }^{a}$ Hydroxyl determination performed by CERIDE (Santa Fé, Argentina). Ash content less than $3 \%$ (w/w).

The lignin potential for polymeric applications have come to be disclosed in the last 50 years, however, only now starts to be considered as a viable and economically competitive material for some companies. According to Gandini (2000), much work still remains to be done in this field in terms of both the optimization of the systems already investigated and the search for new ones. This will be achieved by means of basic, technological and economical research.

FTIR is a widely used technique for polymer characterization and to monitor the polymerization process (see, for example, Elwell et al., 1996; Hua and Dubé, 2001). The objective of this work is to apply the technique of FTIR in the ATR mode, previously developed to study segmented polyurethanes (Barreiro, 2000), to study the formation of lignin based polyurethanes. Our first approach uses lignin as a macromonomer and a linear polyol in the initial formulation, providing flexibility and enabling polymerization in bulk.

\section{Experimental}

\subsection{Materials and formulations}

The lignin-based polyurethanes have been prepared starting with 4,4'-methylene-diphenylene isocyanate (MDI), policaprolactonediol (PCL) of three different average molecular weights (1000, 750 and 400) and a commercial lignin, Indulin AT from Meadwestvaco (South Carolina, USA), at different weight con- tents $(10,15,20$ and $25 \%)$. The characteristics of the chemicals used in the formulations are shown in Table 1. Polycaprolactones, supplied by Solvay Interox (Cheshire, UK) and lignin were degassed overnight under vacuum at $60^{\circ} \mathrm{C}$. MDI (Aldrich) was purified by heating at $60^{\circ} \mathrm{C}$ and filtered through a heated filter just prior to use. Its purity was determined by the standard di-n-butylamine titration and found to be over $99.5 \%$.

Reaction conditions (temperature of $80^{\circ} \mathrm{C}$ and $\mathrm{NCO} / \mathrm{OH}$ ratio of 1) were chosen in order to avoid chemical reticulation due to isocyanate secondary reactions (Lamba et al., 1998).

\subsection{Preparation of the FTIR experiments}

The infrared experiments were conducted on a FTIR Bomen Model MB104 (Quebec, Canada) working in the ATR mode. The ATR accessory from Graseby Specac (Smyrna, GA, USA) was equipped with a heater controller and a silicon crystal, a material optically denser than the polyurethane. In order to obtain a more stable background, the ATR optics was continuously purged with nitrogen during the experiments.

Lignin and PCL were weighed in an analytical balance in the desired proportions and the mixture homogeneized by stirring. Then, molten MDI was added by volume in one portion and the reactive species thoroughly mixed together during $30 \mathrm{~s}$ and rapidly transferred to the ATR cell. Time zero of the reaction was taken as the addition moment of the MDI. The maximum elapsed time between MDI addition and the acquisition of the first scan was $1 \mathrm{~min}$. Three scans per spectrum

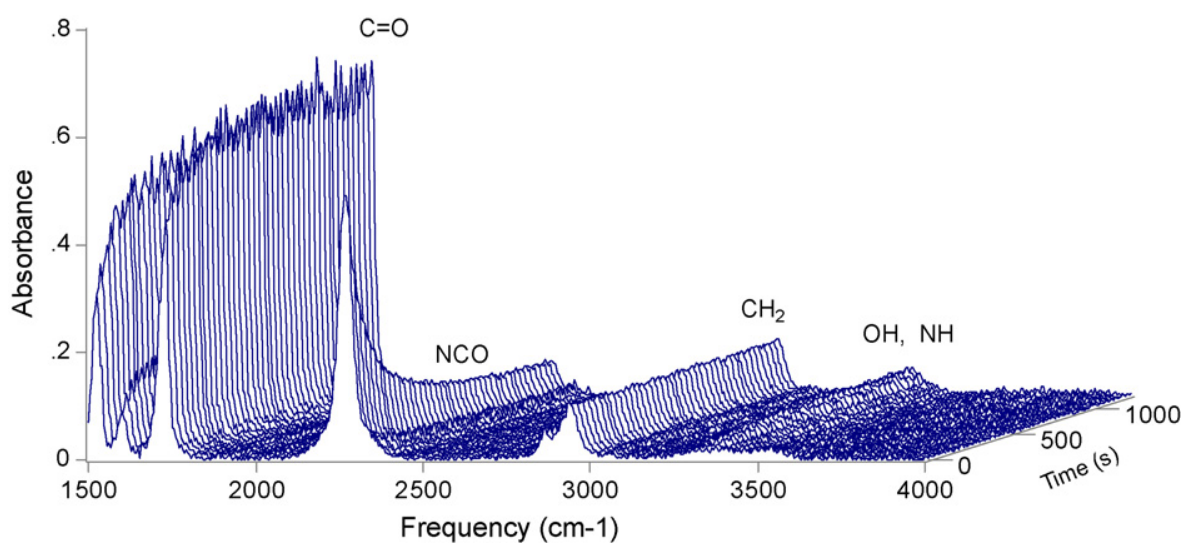

Fig. 1 - Three-dimensional plot of absorbance vs. frequency vs. time for the region $1500-4000 \mathrm{~cm}^{-1}$. The approximate assignments of the most relevant bands are indicated. 

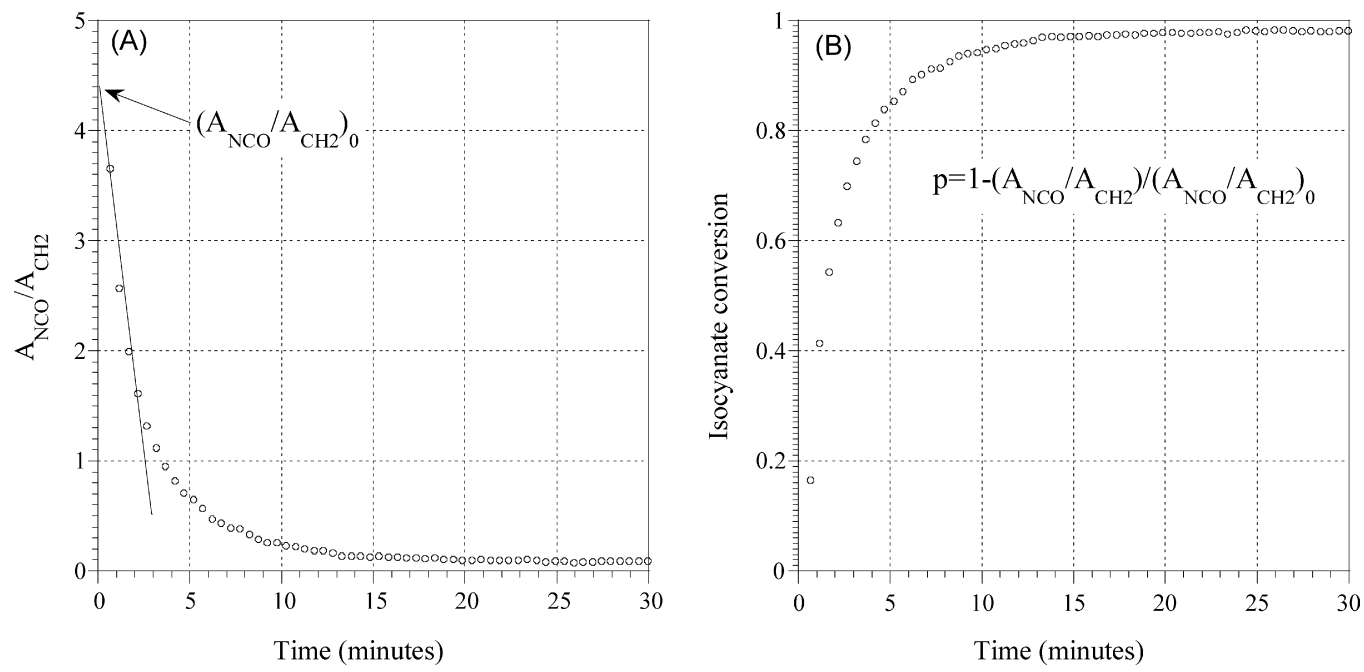

Fig. 2 - Decay in the relative absorbance $\left(A_{N C O} / A_{C H_{2}}\right)(A)$ and the corresponding calculated isocyanate conversion $(p)(B)$. $A_{N C O}$ is the integrated absorbance for the isocyanate group, $A_{\mathrm{CH}_{2}}$ is the integrated absorbance for the $\mathrm{CH}_{2}$ group and $\left(A_{\mathrm{NCO}} / \mathrm{A}_{\mathrm{CH}_{2}}\right)_{0}$ is the relative absorbance extrapolated for time zero.
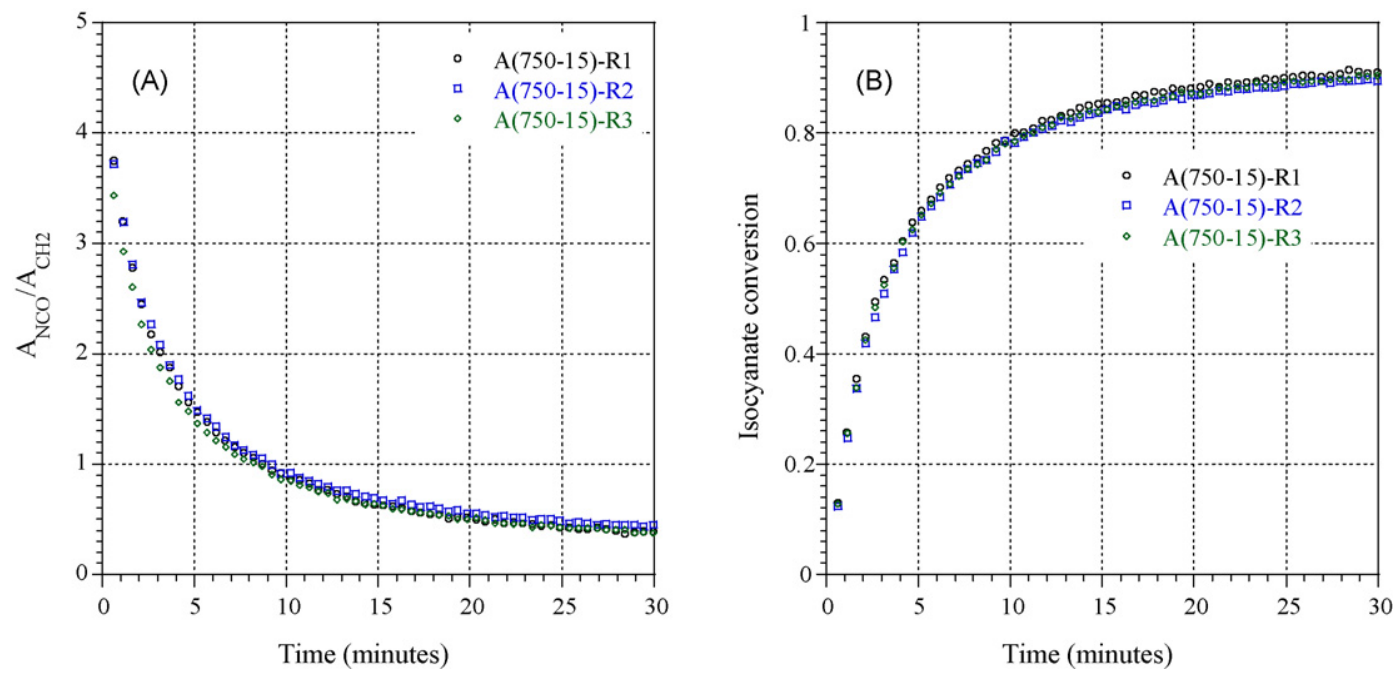

Fig. 3 - Plot showing the three replicas (R1, R2 and R3) for the sample $A(750-15)$ in terms of relative absorbance (A) and the corresponding calculated isocyanate conversion (B).

Table 2 - Summary of the chemical formulations studied

\begin{tabular}{lrcrr} 
Sample & PCL & Lignin content (wt. \%) & Isocyanate conversion (30 min) & Coefficient of variation $^{a}$ (\%) \\
\hline A(400-10) & 400 & 10 & 0.9837 & 0.36 \\
A(400-15) & 400 & 15 & 0.9841 & 0.17 \\
A(400-20) & 400 & 20 & 0.9799 & 0.55 \\
A(400-25) & 400 & 25 & 0.9798 & 0.58 \\
A(750-10) & 750 & 10 & 0.9031 & 1.20 \\
A(750-15) & 750 & 15 & 0.9029 & 1.10 \\
A(750-20) & 750 & 20 & 0.8606 & 1.30 \\
A(750-25) & 750 & 25 & 0.8453 & 0.25 \\
A(1000-10) & 1000 & 10 & 0.8254 & 1.40 \\
A(1000-15) & 1000 & 15 & 0.8059 & 0.42 \\
A(1000-20) & 1000 & 20 & 0.7487 & 0.37 \\
A(1000-25) & 1000 & 25 & 0.7173 & 0.04 \\
\hline
\end{tabular}

a Coefficient of variation: $\mathrm{CV}=\mathrm{s} / \overline{\mathrm{X}} \times 100$ 
were taken at a resolution of $4 \mathrm{~cm}^{-1}$ and the experiment was programmed to record a spectrum every $30 \mathrm{~s}$ during $30 \mathrm{~min}$. Total conversion was never reached to avoid difficulties in removing the sample from the ATR cell, after the experiment. A background file was always collect prior to the execution of an FTIR experiment. GRAMS/32 software (Galatic Industries) was employed for the data acquisition and subsequent data analysis. Fig. 1 shows a typical plot of an FTIR experiment.

\subsection{Swelling tests}

Round samples of approximately $3 \mathrm{~cm}$ of diameter and $3 \mathrm{~mm}$ of thickness were immersed in glass flasks containing dimethyl formamide (DMF) and allowed to stand at ambient temperature during 3 days. After this period of time, the samples were removed from the solvent. Due to experimental difficulties, the final dimensions and consequently the crosslink density were not determined. The main objective of these tests was to confirm that the final sample behaves as a three dimensional polymer network (it swells and do not dissolves in the solvent) and qualitatively evaluate the release of free lignin.

\section{Results and discussion}

\subsection{Analysis of the FTIR experiments}

The isocyanate absorption band is assigned at approximately $2270 \mathrm{~cm}^{-1}$ and the decay in the intensity of this absorbance can be used to monitor the isocyanate group conversion during the polymerization. Quantification is based on the Beer-Lambert law and the validity for the isocyanate absorption is well established in literature (Boufi et al., 1993; Fan et al., 1996; Elwell et al., 1996) and confirmed in the previous work (Barreiro, 2000). In order to correct thickness changes during polymerization, a ratio between the absorbance of the isocyanate group and that of an internal standard (a group whose concentration does not change during the reaction course) is calculated. In this study, the absorbance corresponding to the $\mathrm{CH}_{2}$ stretching region, assigned at approximately $2870 \mathrm{~cm}^{-1}$, was used. Fig. 2 shows the data extracted from an FTIR experiment and the corresponding calculated isocyanate conversion curve. All the experiments were done in triplicate. Fig. 3 shows the three replicas of the experiment performed with MDI, PCL750 and Indulin AT at a weight content of 15\%. The conversion achieved at 30 min was 0.9020 with a coefficient of variation of $1.1 \%$. Table 2 summarizes the results obtained for all the synthesized samples. For this set of experiments, the coefficient of variation never exceeded $1.4 \%$, confirming the reproducibility of the experiments.

\subsection{Lignin reactivity}

Inspection of lignin reactivity with isocyanates was performed by comparing the experimentally determined isocyanate conversion with a reference conversion, calculated on the basis of total consumption of the polyol hydroxyls. This conversion was designated by critical conversion. Since reaction conditions were chosen in order to avoid isocyanate secondary
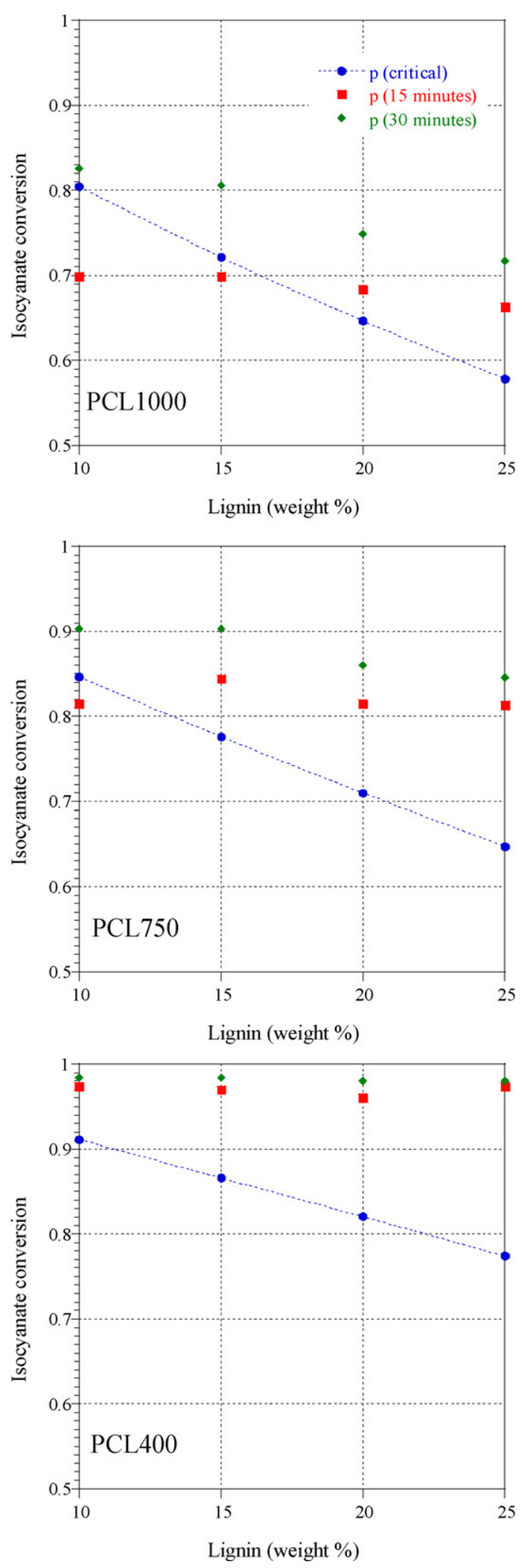

Fig. 4 - Comparison between critical ( $p$ (critical)) and experimental isocyanate conversion $(p(15 \mathrm{~min})$ and $p(30 \mathrm{~min}))$ as a function of lignin content for the series PCL1000, PCL750 and PCL400. 
reactions, the achievement of conversions higher than the critical one pointed out for the incorporation of lignin through chemical reaction, i.e., formation of urethane linkages due to the reaction of lignin hydroxyls with isocyanates. As can be observed by the analysis of Fig. 4, lignin incorporation was more effective for the series PCL400 and less effective for the series PCL1000. These conclusions were also supported by the performed swelling tests. By visual inspection, the observed swelling volume was lower for samples synthesized with PCL400 (higher reticulation) and higher for samples synthesized with PCL1000 (lower reticulation). Moreover, free lignin release was more noticeable for samples synthesized with PCL1000 and almost absent in samples synthesized with PCL400. Fig. 5 shows the polyurethane samples with $20 \%$ lignin after being swollen in DMF. The sample synthesized with PCL1000 shows an appearance of a "gel-like" material with a poorly defined shape; the sample with PCL750 is an elastic material and the sample with PCL400 is a brittle material.

\subsection{Preliminary kinetic studies}

In a first attempt to model kinetics, the experimental data were inspected according to a global second order model. A simplification was considered assuming equal reactivity for all hydroxyls presented in the reaction mixture. This procedure is widely used to model complex polyurethane systems, for example, to model the synthesis of polyurethanes from suberin (Cordeiro et al., 1997), from oxypropylated cork (Evtiouguina et al., 2001), and from oxypropylated sugar beet pulp (Pavier and Gandini, 2000). This approach was also used to model the synthesis of segmented polyurethane systems where more than one type of hydroxyl is present (Barreiro, 2000).

Under stoichiometric conditions, i.e. $[\mathrm{NCO}]_{0}=[\mathrm{OH}]_{0}$, the following classical expression is obtained:

$\frac{1}{1-p}=1+k[\mathrm{NCO}]_{0} \mathrm{t}$

where $p$ represents the isocyanate conversion, $[\mathrm{NCO}]_{0}$ the initial concentration of isocyanate groups, $t$ the time, and $k$ the rate constant.

Fig. 6 shows the typical second order plots $(1 /(1-p)$ versus time) for the performed experiments. The corresponding linear fits are represented, and the values of isocyanate conversions up to where linear fits are valid are stressed out. These conversions were calculated assuming a determination coefficient $\left(R^{2}\right)$ greater than 0.99 . For the series PCL1000 and PCL750 with a lignin content of $10 \%$ and $15 \%$, the second order treatment proved to be adequate. For the same series with $20 \%$ and $25 \%$, the model was always good up to an isocyanate conversion of 0.63 (PCL1000, 25\% lignin), 0.67 (PCL1000, 20\% lignin), 0.78 (PCL750, 25\% lignin), and 0.83 (PCL750, 20\% lignin). After these conversions, a negative deviation is observed and becomes more pronounced with the increasing of lignin content and decreasing of polyol molecular weight. For all the series with PCL400, a positive deviation is observed since the early stages of the polymerization reaction. This phenomenon has been reported for other polyurethane systems

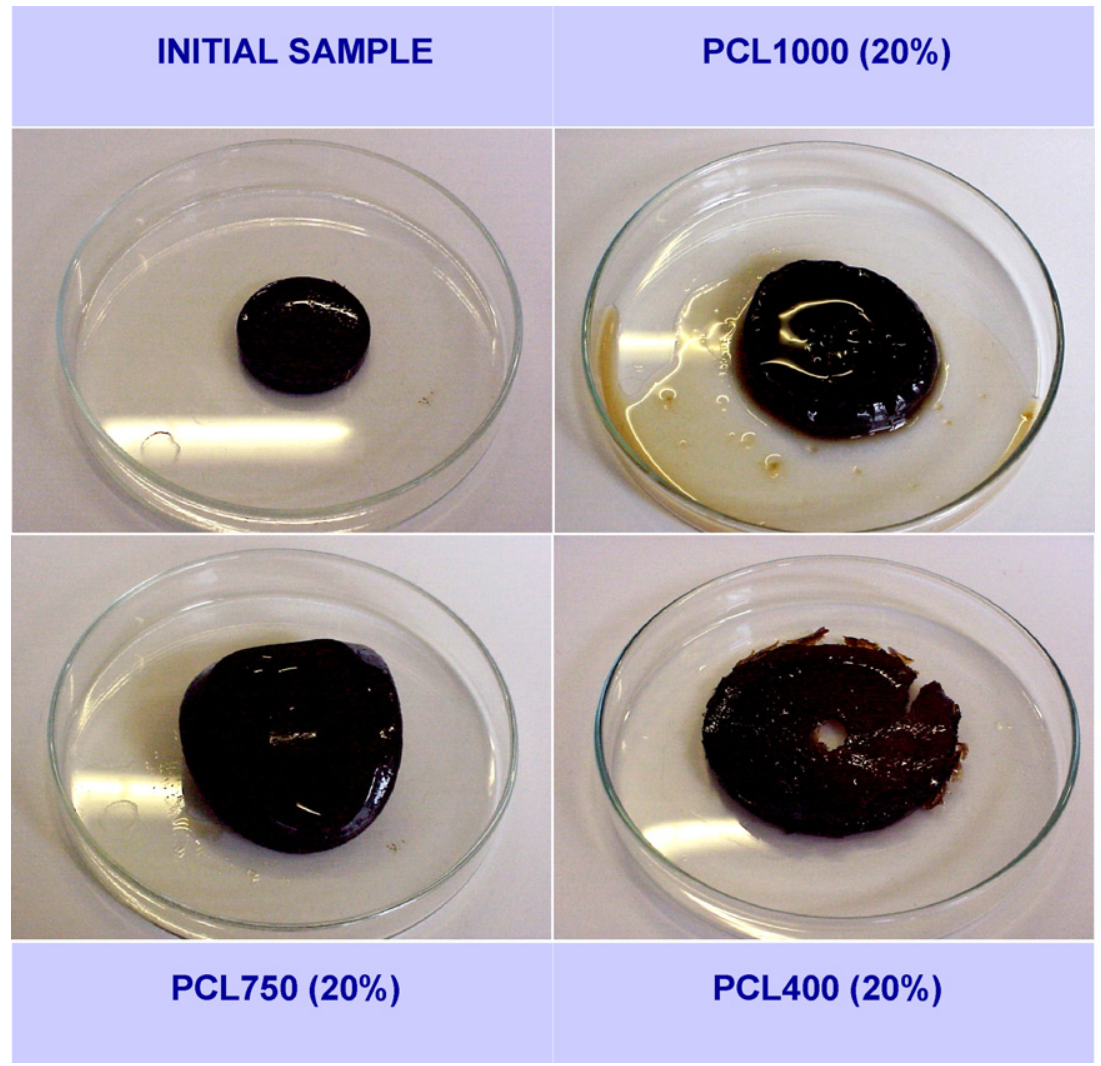

Fig. 5 - Polyurethane samples synthesized with $20 \%$ of lignin and different molecular weight polycaprolactones (PCL1000, PCL750 and PCL400) after being swollen in DMF. One initial sample is included as a reference. 

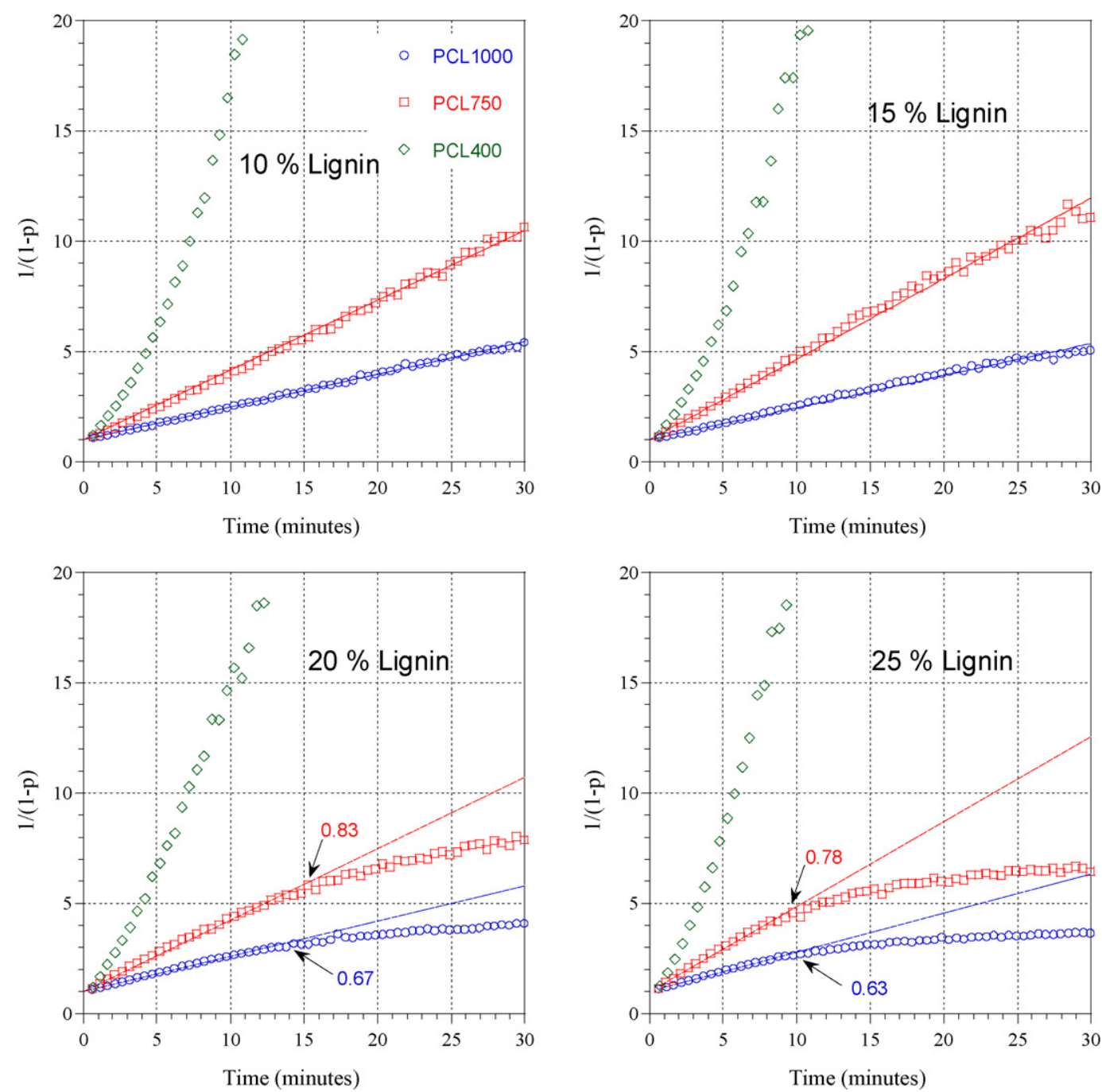

Fig. 6 - Second-order plots for the chemical system PCL, MDI and Indulin AT: effect of lignin content (10, 15, 20 and 25\%) and polycaprolactone molecular weight (PCL1000, PCL750 and PCL400). Note the isocyanate conversion at which negative deviation begins.

and was ascribed to an autocatalytic effect of the forming urethane group (Hager et al., 1981). The occurrence of isocyanate secondary reactions can also justify these deviations, as suggested by Cordeiro et al. (1997), Pavier and Gandini (2000), and Evtiouguina et al. (2001). Nevertheless, the later should not be applied to this work since the experimental conditions were chosen in order to avoid secondary reactions. The confirmation of effective lignin incorporation, as discussed in Section 3.2, also corroborates this conclusion.

The present work is intended to be a simplified approach and a deeper understanding of the kinetics of these complex chemical systems needs further work by performing complementary kinetic studies with lignin model compounds.

\section{Conclusions}

(i) In the present work, the technique of FTIR-ATR was successfully applied to the study of lignin based polyurethanes. The methodology used was presented and the reproducibility of the experiments demonstrated. This methodology could provide a valuable tool to study and optimize a chemical formulation at a very small scale (3-5 g).

(ii) The results achieved within this work pointed out for lignin incorporation, in the final three component polyurethane sample, through chemical reaction with isocyanates, i.e., formation of urethane linkages. Final conversion, determined experimentally by FTIR-ATR, was always greater than the defined critical conversion. Swelling tests also confirm this observation.

(iii) The chemical system chosen is of crucial importance considering lignin incorporation and final properties of the produced polyurethane sample. The use of a low molecular weight polyol favours lignin incorporation but is too short to provide flexibility to the final product.

(iv) The assessment of kinetic data from the FTIR-ATR experiments is also demonstrated. Although some of the results presented here could only be considered preliminary, 
the potentialities of the FTIR-ATR technique were fully demonstrated.

\section{Acknowledgements}

The authors wish to thank Solvay (Cheshire, UK) and Meadwestvaco (South Carolina, USA) for kindly providing the polycaprolactone and lignin raw materials, respectively. Financial support from the Portuguese Foundation for Science and Technology (grant SFRH/BD/18415/2004) is acknowledged. Lignin characterization performed by Dra. Susana Gervasio, from CERIDE (Santa-Fé, Argentina), is also acknowledged.

\section{REFERENCES}

Barreiro, M.F., 2000. Formação e Estrutura dos Poliuretanos Segmentados. PhD Thesis. FEUP, Porto, Portugal.

Boufi, S., Belgacem, M.N., Quillerou, J., Gandini, A., 1993. Urethanes and polyurethanes bearing furan moieties. 4: Synthesis kinetics and characterization of linear polymers. Macromolecules 26, 6706-6717.

Cordeiro, N., Belgacem, M.N., Gandini, A., Pascoal Neto, C., 1997. Urethanes and polyurethanes from suberine: 1 Kinetic study. Ind. Crops Prod. 6, 163-167.

Elwell, M.J., Ryan, A.J., Grünbauer, H.J.M., Van Lieshout, H.C., 1996 An FT-IR study of reaction kinetics and structure development in model flexible polyurethane foam systems. Polymer 37 (8), 1353-1361.

Evtiouguina, M., Gandini, A., Pascoal Neto, C., Belgacem, M.N., 2001. Urethanes and polyurethanes based on oxypropylated cork: 1. Appraisal and reactivity of products. Polym. Int. 50, 1150-1155.

Fan, L.H., Hu, C.P., Zhang, Z.P., Ying, S.K., 1996. Polymerization kinetics of polyurethane and vinyl ester resin interpenetrating polymer networks by using Fourier transform infrared spectroscopy. J. Appl. Polym. Sci. 59, 1417-1426.

Gandini, A., 2000. Les polymères naturels: structure, modifications et applications. VI. Les lignines et leur utilisation dans les matériaux macromoléculaires. GFP, Paris, pp. 159-196.

Gandini, A., Belgacem, M.N., 1998. Recent advances in the elaboration of polymeric materials derived from biomass components. Polym. Int. 47, 268-276.

Glasser, W.G., Sarkanen, S. (Eds.), 1989. Lignin Properties and Materials. ACS Symposium Series 397. ACS.

Hager, S.L., MacRury, T.B., Gerkin, R.M., Critchfield, F.E., 1981. Urethane block polymers. Kinetics of formation and phase development. Urethane Chemistry and applications. Am. Chem. Soc. Symp. Ser. 172, 149-166.

Hu, T.Q. (Ed.), 2002. Chemical Modification, Properties and Usage of Lignin. Kluwer Academic/Plenum Publishers, New York.

Hua, H., Dubé, A., 2001. Terpolymerization monitoring with ATR-FTIR spectroscopy. J. Polym. Sci. Polym. Chem. 39, 1860-1876.

Lamba, N.M.K., Woodhouse, K.A., Cooper, S.L., 1998. The chemistry of polyurethane copolymers. In: Polyurethanes in Biomedical Applications. CRC Press, Boca Raton, pp. 5-25 (Chapter 2).

Meister, J.J., Chen, M., Chang, F., 1992. Make polymers from biomass. Chemtech, 430-435.

Pavier, C., Gandini, A., 2000. Urethanes and polyurethanes from oxypropylated sugar beet pulp I. Kinetic study in solution. Eur. Polym. J. 36, 1653-1658. 